\title{
Correction to: iHIVARNA phase lla, a randomized, placebo-controlled, double- blinded trial to evaluate the safety and immunogenicity of iHIVARNA-01 in chronically HIV-infected patients under stable combined antiretroviral therapy
}

\author{
Wesley de Jong ${ }^{1}$, Joeri Aerts², Sabine Allard ${ }^{3}$, Christian Brander ${ }^{4,5,6,7}$, Jozefien Buyze ${ }^{8,9}$, Eric Florence ${ }^{8,9}$, \\ Eric van Gorp ${ }^{1}$, Guido Vanham ${ }^{8,9}$, Lorna Leal ${ }^{10,11}$, Beatriz Mothe ${ }^{4,7}$, Kris Thielemans ${ }^{2}$, Montse Plana ${ }^{10}$, \\ Félipe Garcia ${ }^{10,11^{*}}$, Rob Gruters ${ }^{1 *}$ and on behalf of the iHIVARNA consortium
}

\section{Correction to: Trials}

https://doi.org/10.1186/s13063-019-3409-1

Following publication of the original article [1], we have been notified that the end note would need to be adjusted.

Originally published note:

- During the revision process of this paper, it became apparent that the study product iHIVARNA-01 contains an error in that the RNA sequence contained by mistake a second start codon in front of the HTI immunogen coding sequence. This error is likely to influence the expression of the HTI protein, from the mRNA vaccine. Even though the degree to which this impacted the expression of HTI remains unclear, the results of the preclinical trial show that there is sufficient expression for the induction of an immunogen-specific $\mathrm{T}$-cell response in mice.
The original article can be found online at https://doi.org/10.1186/s13063019-3409-1

*Correspondence: fgarcia@clinic.cat; r.gruters@erasmusmc.nl

${ }^{10}$ Institut d'Investigacions Biomèdiques August Pi i Sunyer (IDIBAPS),

Villarroel, 170, 08036 Barcelona, Spain

'Department of Viroscience, Erasmus MC, Room Ee-1726, P.O. Box 2040

3000, CA, Rotterdam, The Netherlands

Full list of author information is available at the end of the article
Corrected end note:

- After completing the phase I and phase II trial, it became apparent that the study product iHIVARNA-01 contains an error in that the RNA sequence contained by mistake a second start codon upstream of the HTI immunogen coding sequence. This error is likely to influence the expression of the HTI protein, from the mRNA vaccine. The degree to which this impacted the expression of HTI remains unclear. The results of the preclinical study showed an induction of an immunogen specific $\mathrm{T}$ cell response in mice which could not be correlated with known HTI expression as the expression level of the HTI peptide has not been assessed. Similarly, in the clinical studies, the level of expression of the immunogen was not quantified. 
of Biomedical Sciences, University of Antwerp, Antwerp, Belgium. ${ }^{10}$ Institut d'Investigacions Biomèdiques August Pi i Sunyer (IDIBAPS), Villarroel, 170, 08036 Barcelona, Spain. ${ }^{11}$ Infectious Diseases Unit, Hospital Clínic, Villarroel, 170, 08036 Barcelona, Spain.

\section{0.}

\section{Reference}

1. Jong, et al. iHIVARNA phase lla, a randomized, placebo-controlled, doubleblinded trial to evaluate the safety and immunogenicity of iHIVARNA-01 in chronically HIV-infected patients under stable combined antiretroviral therapy. Trials. 2019;20:361. https://doi.org/10.1186/s13063-019-3409-1. 